\title{
Markers of Apoptosis and Phagocytosis Suggest a Function for Maternal Macrophages in Uterine Remodeling during Mid-Pregnancy and Postpartum
}

\author{
Gargi Mishra ${ }^{1}$, Becca Ortiz², Ledia Gebremedhin², Kristina Ramsden², Anh Nguyen², Sarah Bacon² \\ 1. Department of Biochemistry, Mount Holyoke College, South Hadley, MA, USA \\ 2. Department of Biological Sciences, Mount Holyoke College, South Hadley, MA, USA
}

Humans and rodents possess the most invasive form of placentation characterized by a highly vascularized and intimate connection between a mother and the fetus. For this connection to form, extraembryonic cells called trophoblasts penetrate the uterus reaching to the inner third of the myometrium to assist natural killer (NK) cells in the remodeling of spiral arteries, the vessels that supply blood to the endometrial lining of the uterus. The spiral arteries lose their smooth muscle wall, dilating to become low-pressure high-volume conduits for blood supply to the fetus [1]. Remodeling of spiral arteries is important for a successful pregnancy and different immune cells, especially NK cells and macrophages, appear to play a role [2].

While the role of NK cells in arterial remodeling has been well-studied and established, the role of macrophages still remains unclear ${ }^{2}$. We analyzed rat metrial gland (a model for the human deep placental bed) and decidual tissue from gestational days (GD) 12, 14, 16 and postpartum day (PPD) 3 using quantitative real-time PCR (data not shown). We found high levels of mRNA corresponding to the rat pan-macrophage marker CD68 during mid-to-late pregnancy and postpartum time points. Phosphatidylserine receptor MerTK mRNA was expressed at modest levels during mid-pregnancy and rose during postpartum. This led us to hypothesize that MerTK might be expressed by macrophages, implying a role for macrophages in clearing apoptotic cells (ACs) during pregnancy and postpartum.

To test our hypothesis further, we performed double immunofluorescence staining across pregnancy (GD12 and GD16) and postpartum (PPD1 and PPD3) time points in the rat using OCT-embedded frozen uterine tissue sections (6um thick) containing metrial gland and decidual regions. We performed CD68 and Annexin $\mathrm{V}$ co-immunostaining to ascertain the proximity between macrophages and apoptotic cells (ACs); CD68 and MerTK co-immunostaining to see if macrophages were expressing MerTK; and CD68 and Perforin co-immunostaining to see if there was any cross-talk between macrophages and natural killer cells. Due to technical difficulties while cryosectioning, we were only able to stain decidua on GD12 and PPD1, and metrial gland on GD16 and PPD3. We also stained virgin endometrium. Each tissue time point was represented by one animal $(n=1)$.

We found that $\mathrm{CD}^{+}$cells were near Annexin $\mathrm{V}^{+}$(apoptotic) cells across all 5 time points studied (figures 1a-1e). This suggested that macrophages might be involved in engulfing ACs via binding to phosphatidylserine moieties exposed on the surface of ACs. CD68 and MerTK colocalized to the same cell type in the decidual region on GD12 (figure 2b) and PPD1 (figure 2d), while in the metrial gland on GD16 (figure 2c) and PPD3 (figure 2e), CD68 and MerTK did not colocalize. MerTK was also expressed by other unknown cell types (figures 2a-2e). This suggests that macrophages in the metrial gland region do not express MerTK on GD16 and PPD3, but due to lacking data, no conclusions can be made for the macrophage phenotype in the metrial gland on GD12 and PPD1 or in the decidua on GD16 and PPD3. Macrophages and perforin ${ }^{+}$NK cells usually appear to be present in distinct regions of the tissue (figures 3a-3e). For instance, macrophages can be seen in the lumen of vessels and perforin ${ }^{+} \mathrm{NKs}$ 
are mainly seen interspersed within the metrial gland's or decidua's tissue architecture. This is particularly apparent in the decidual tissue on PPD1 (figure 3d) and metrial gland region on PPD3 (figure 3e). Moreover, in the metrial gland region on GD16, perforin ${ }^{+}$NK cells appear in high spatial proximity to $\mathrm{CD}^{+} 8^{+}$macrophages (figure 3c). These perforin ${ }^{+} \mathrm{NK}$ cells in the metrial gland on GD16 might be granulated metrial gland (GMG) cells. This proximity between $\mathrm{CD}^{+} 8^{+}$and perforin ${ }^{+}$cells suggests a possible cross-talk between macrophages and GMG cells.

In conclusion, uterine macrophages appear to be heterogeneous with regard to MerTK expression. NK cells might also express MerTK, so it is important to do co-labelling experiments involving an NK cell receptor (such as CD161) and MerTK. More automated imaging methods will enable us to quantify different populations. But this pilot study showed us that some macrophages do express MerTK, and that this may have functional significance to the role that macrophages play in uterine remodeling [3].

\section{References:}

[1] Burton, G. et al, Placenta. 30 (2009), p. 473.

[2] Faas, M. M., and Vos, P. D. Placenta. 56 (2017), p. 44.

[3] The authors acknowledge funding from the Microscopy Society of America. Blanca CarbajalGonzalez is thanked for her many useful contributions to this work.
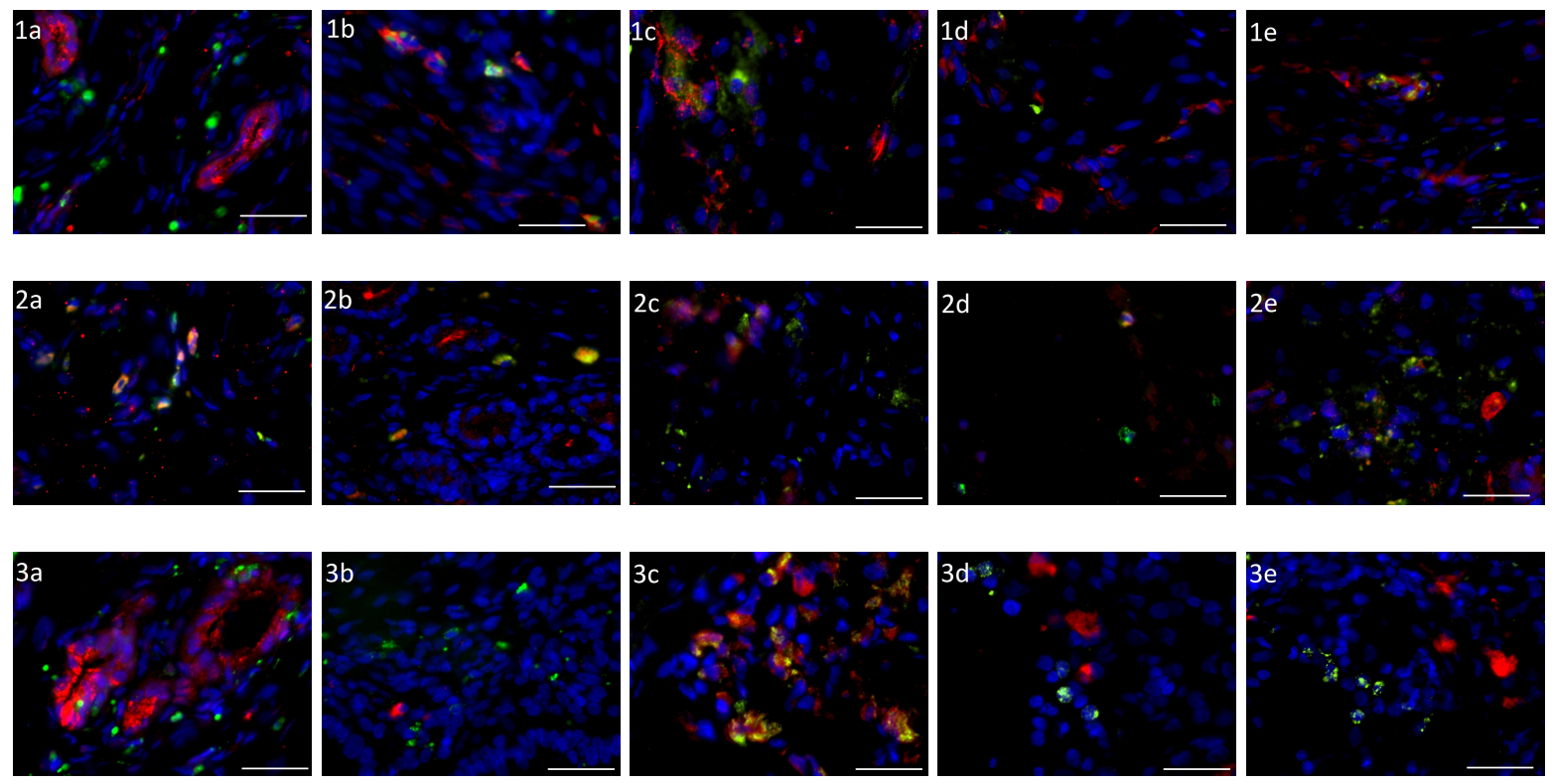

Figure 1. 40X magnification merged images of rat uterine tissue. CD68 is green and Hoechst is blue in each panel. Panels labeled (left to right) 1a through 1e refer to CD68 and Annexin V (red) double immunofluorescence staining; 2a through 2e refer to CD68 and MerTK (red) double immunofluorescence; 3a through 3e refer to CD68 and Perforin (red) double immunofluorescence. Alphabet portions of the panel labels refer to the following tissue sections (left to right): a, Virgin decidua; b, GD12 decidua; c, GD16 metrial gland and placenta; d, PPD1 decidua; and e, PPD3 metrial gland. Scale bar represents 50um. 